\title{
Die pure Eleganz \\ der Mathematik
}

Wie allgemein bekannt, unterscheiden sich Mathematiker erheblich von der Mehrheit ihrer Mitmenschen. Man braucht nur einige von Enzensbergers Gedichten zu lesen, in denen er staunend feststellt, dass sie (die Mathematiker) sich vornehmlich in gekrümmten Räumen aufhalten und ohne weiteres Links- mit Rechtsidealen vertauschen, ganz zu schweigen von Unterkörpern, die für sie etwas ganz anderes bedeuten, als der unbefangene Beobachter meinen könnte.

Hier wollen wir uns mit einem weiteren Unterschied befassen, der geradezu mitten ins Leben greift. Während sich die Mehrheit über die wirklich wichtigen Dinge wie Mülltrennung oder Zahnpflege einig ist, sich aber über künstlerische Dinge lustvoll auseinander setzt (der Volksmund sagt bekanntlich: Über Geschmack lässt sich nicht streiten - und meint natürlich das Gegenteil), so ist das in der mathematischen Welt genau umgekehrt. Spätestens seit Plato stehen sich Platonisten und Formalisten

\footnotetext{
"[...] es ist durchaus kein Zufall, dass den meisten Mathematikern ästhetische Kriterien nicht fremd sind. Es genügt ihnen nicht, dass ein Beweis stringent ist; ihr Ehrgeiz zielt auf , Eleganzr." H.M. Enzensberger
}

ideologiebewehrt gegenüber; der Disput, ob mathematische Gesetze entdeckt oder erfunden werden, ist ein Dauerbrenner; und die Frage über den Primat von reiner versus angewandter Mathematik kann ganze Institute in Aufregung versetzen. Aber wenn sich zwei Mathematiker über ein Blatt Papier beugen und der eine sagt: »Das ist ein ausgesprochen eleganter Beweis!«, kann er sich der Zustimmung seines Kollegen sicher sein. Über Schönheit und Eleganz von mathematischen Formeln, Sätzen und insbesondere Beweisen gibt es keinen Disput, da sind sich alle einig.

Aber was ist nun Eleganz in der Mathematik? Merkwürdigerweise lässt sich darüber bei den Großschreibern 


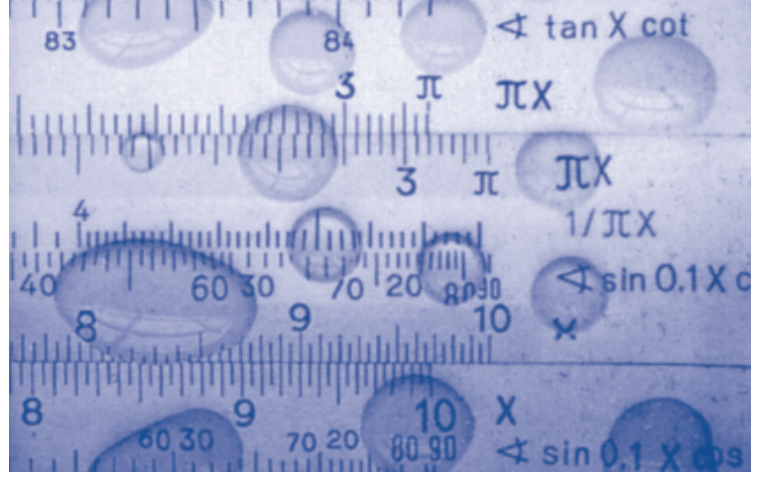

der Zunft nichts erfahren (wohl aber über Schönheit, wie wir gleich sehen werden). Ich will die Leser ein wenig in die mathematische Welt verführen, eine ganz persönliche Definition von Eleganz vorschlagen und sie mit einigen klassischen Beispielen illustrieren.

\section{Vom Schönen und Wahren}

Als 1995 Andrew Wiles das berühmteste offene mathematische Problem, die Fermat'sche Vermutung, bewies, war dies allen Zeitungen eine Schlagzeile auf Seite eins wert. In einer überregionalen Zeitung stand zu lesen: »Rechenkünstler aus Cambridge löste 350 Jahre altes mathematisches Rätsel«. Die virtuose Abfolge von logischen Schlüssen und Strukturaussagen in der Arbeit von Wiles als Rechnen zu bezeichnen könnte der Wahrheit nicht ferner sein. Aber offenbar ist es allgemeine Ansicht, dass ein Mathematiker jemand ist, der 99 Formeln durcheinander mischt und daraus eine 100. Formel gebiert.

Was einige der größten Mathematiker dazu meinen, hört sich anders an. Aristoteles schreibt in seiner Metaphysik: "Insbesondere die mathematischen Wissenschaften drücken Ordnung, Symmetrie und Beschränkung aus - und dies sind die höchsten Formen der Schönheit. «Johannes Kepler, der ohnehin zum Schwärmen neigte, war hingerissen von den sgoldenen Proportionen der Mathematik. Henri Poincaré postulierte den erstaunlichen Satz: „Das Ästhetische mehr als das Logische ist die dominierende Komponente in der mathematischen Kreativität." G. H. Hardy, ein Meister der Zahlentheorie, die notorisch komplizierte Formeln hervorbringt, merkte mit untypischem britischen Overstatement an: »There is no permanent place for ugly mathematics!« Ich hoffe, Ihre Sicht des Mathematikers als rigorosem Rechenmeister endgültig erschüttert zu haben, wenn ich den Physiker Paul Dirac zitiere: »Es ist wichtiger, dass eine Gleichung schön ist, als dass sie mit dem Experiment übereinstimmt."Am prägnantesten beschreibt diese Gegenwart des Schönen vielleicht Hadamard in seiner Psychology of Invention: „Das mathematische Genie offenbart sich in zwei Weisen; es wählt mit untrüglicher Sicherheit unter einer Vielzahl von Alternativen die einzig richtige, und es wird dabei geleitet von der Idee des Vollkommenen, einer Ahnung vom Paradies, vom ewig Gültigen.«

Man sollte nun nicht meinen, die Mathematiker hät- ten sich in ihren Meta-Schriften nur mit dem Schönen beschäftigt - ganz im Gegenteil. In der Hauptsache geht es um Mathematik als Denkmodell, als Abbild der Wirklichkeit, kurz: um Erkenntnis und Wahrheit. Derselbe Poincaré schreibt in seinen Letzten Gedanken sinngemäß: Wissenschaft ist der Drang nach Wahrheit auf sittlicher Basis. Wittgenstein betont die kategorische Strenge der Logik, und Popper führte das masochistische Prinzip in die Wissenschaft ein: Eine Theorie ist nur dann etwas wert, wenn sie falsifizierbar ist. Man liest Sätze wie: »Nur der Nutzen adelt die Erkenntnis«, »Mathematik ist $\mathrm{Hu}$ manismus«, und in dem vielleicht besten neueren Buch Erfahrung Mathematik von Davis und Hersh sind ganze vier von 400 Seiten Fragen der Ästhetik gewidmet.

Auch wenn der ästhetische Aspekt in den Hintergrund tritt, scheint er ein einigendes Band zu sein. Wenn ein Gutachter die bedeutenden Anwendungen einer mathematischen Arbeit herausstellt, so wird der Autor erfreut sein; schreibt er aber in seinem Referat: "The beauty of the theorem is matched by the elegance of its proof «, so kann er sich der Rührung und des ewigen Dankes des Verfassers sicher sein.

\section{Das Buch der Beweise}

Legenden werden üblicherweise nach dem Tod gestrickt. Entstehen sie zu Lebzeiten, so muss es sich um einen außergewöhnlichen Menschen handeln - und der ungarische Mathematiker Paul Erdös war solch eine Jahrhunderterscheinung. Er war der produktivste Mathematiker der jüngeren Geschichte mit über 1500 Veröffentlichungen. Unermüdlich reiste er von Kontinent zu Kontinent, die eine Woche in Jerusalem, dann in den USA, und nächsten Monat war ein Touch-down in Berlin fällig. Mit seinem einen Koffer in der Hand war seine Begrüßung zugleich sein Motto: »My brain is open."Gleichermaßen großzügig im Leben wie in der Wissenschaft, verschenkte er nicht nur die meisten seiner vielen Preisgelder, sondern teilte auch seine Ideen und Geistesblitze mit jedem, der sie hören wollte. Er lebte für die und in der Mathematik. Es gibt zahllose Anekdoten über Erdös, die folgende hat den Vorzug, wahr zu sein, weil ich selber dabei war. Eines Abends gegen 22 Uhr saßen wir zu dritt in New York über einem Problem, besser gesagt: Wir saßen fest und kamen nicht weiter. Plötzlich sagte Erdös: „Am besten, 


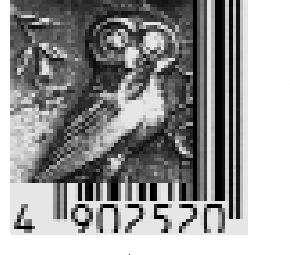

$12 \mid \mathbf{1 3}$

\section{Ein Computer-Berweis ist weder transparent noch von \\ Leichtigkeit beseelt, aber vor allem lehrt er uns nichts.}

wir rufen meinen Freund Davenport in Cambridge an, der kann uns bestimmt weiterhelfen.«-»Aber«, so wandte ich ein, »in Cambridge ist es jetzt 4 Uhr morgens.« Darauf Erdös: »Na umso besser, dann ist er sicher zu Hause."

Ein immer wiederkehrendes Diktum von Paul Erdös führt geradewegs zu unserem Thema. Manche Beweise, erzählte er, sind wunderschön, sie haben nur den kleinen Makel, dass sie falsch sind. Wieder andere sind richtig, aber hässlich. Aber, so war er überzeugt, für jeden mathematischen Satz gibt es den Beweis, und mehr noch: Es gibt das BUCH, in dem der liebe Gott die perfekten Beweise aufbewahrt. Und er fügte hinzu: »Man muss nicht an Gott glauben, aber als Mathematiker sollte man an die Existenz des Buches glauben. « Mitte der neunziger Jahre schlugen Günter Ziegler und ich ihm vor, gemeinsam eine erste (und sehr bescheidene) Annäherung an das BUCH aufzuschreiben. Erdös hat die Idee enthusiastisch aufgegriffen, die Fertigstellung von Proofs from the $B O O K$ aber nicht mehr erlebt. Wahrscheinlich wäre er weniger als wir über die überwältigende Resonanz auf das BUCH überrascht gewesen. Mathematiker sind in der Mehrzahl introvertierte Menschen. Die ungezählten Zuschriften, die wir erhielten, mit Vorschlägen, Korrekturen, eigenen Erlebnissen und viel Zustimmung, kommen daher einem veritablen Gefühlsausbruch gleich. Das BUCH hatte offenbar eine Saite zum Schwingen gebracht, die jedem mathematischen Instrument zu Eigen ist - mit Eleganz als gemeinsamer Grundstimmung.

\section{Die Leichtigkeit des Augenblicks}

Das Wesen der Mathematik ist das Beweisen von Sätzen - und das ist es, was Mathematiker tun: Sie beweisen Sätze. Aber, um ehrlich zu sein, was sie wirklich beweisen wollen, wenigstens einmal in ihrem Leben, ist ein Lemma, wie das Lemma von Fatou in der Analysis oder das von Gauß in der Zahlentheorie. Mit fast jedem berühmten Namen ist solch ein Lemma verbunden - das Wort hat für Mathematiker-Ohren einen fast mythischen Klang.

Nun, wann wird eine mathematische Aussage ein wirkliches Lemma? Zunächst sollten die Aussage (und der Beweis) vollkommen transparent sein: Das Komplexe wird einfach und folgerichtig, und man weiß: That's it!
Auch ein akuter Anfall des Livor academicus (einer besonders häufigen Form des gemeinen Neides) ist denkbar: Warum habe ich das nicht gesehen? Zweitens sollte der Satz stringent sein (oder im mathematischen Jargon: tief). Der Beweis zeigt auf, worauf es wirklich ankommt; er hat vielfältige Anwendungen, sogar auf Probleme, die nichts miteinander zu tun zu haben scheinen. Und schließlich sollte ein Moment der Leichtigkeit vorliegen. Jeder Wissenschaftler hat einen Sack voll Methoden, den er immer wieder öffnet (von manchen, auch prominenten, heißt es, sie hätten mit nur einer Idee ganze Disziplinen beherrscht). Aber es gibt diese Ausnahmekönner, die ein Problem der Algebra mit Methoden der Topologie lösen und umgekehrt, die uns zeigen, dass die Mathematik in all ihrer Vielfalt eine wunderbare Einheit bildet, die uns die Leichtigkeit des Denkens für einen Augenblick erahnen lässt. Transparenz, Stringenz und Leichtigkeit bilden also den Dreiklang eines eleganten Beweises.

Als 1976 das berühmte und seit 100 Jahren offene Vier-Farben-Problem mit enormem Computereinsatz gelöst wurde, zog dies eine bis heute andauernde Kontroverse nach sich: Ist solch ein Beweis akzeptabel? Ein computergestützter Beweis derartiger Länge ist für den, der nicht über ähnliche Rechnerkapazität verfügt, nicht nachzuprüfen. Er ist, überspitzt formuliert, mehr eine Glaubenssache als ein mathematisches Faktum. Solche Einwände sind heute, auch aufgrund gestiegener Rechenleistung, nicht wirklich relevant. Der Computer macht nichts anderes als die Mathematiker mit Bleistift und $\mathrm{Pa}-$ pier: Er geht Schritt für Schritt voran, bis das Resultat vorliegt. Aber das ist genau der springende Punkt: Ein Computer-Beweis ist weder transparent noch von Leichtigkeit beseelt, aber vor allem lehrt er uns nichts. Er zerhackt das Problem in endlich viele Einzelfälle und schließt dann einen Fall nach dem anderen aus, kurz: Er erschlägt den Satz, statt ihn zu erklären.

Der Physiker Eugene Wigner wird oft mit dem Wort von der "unreasonable effectiveness of mathematics« zitiert. Bescheidener drückt dies Erwin Schrödinger aus: "Ob die Natur nach mathematischen Gesetzen funktioniert, wissen wir nicht, aber wir haben vorläufig nichts Besseres.«Ich möchte Wigners Bonmot mit dem Satz von der sunreasonable beauty of mathematics ergänzen. »Die schöne Formel ist oft nahe an der wahren Natur, 
\&

B
4
$10_{0}^{0} 0^{1}$

(1)
3
7
(3) 2 und der elegante Beweis ist oft auch derjenige, der uns den größten Erkenntnisgewinn beschert.«

\section{Vom Unendlichen ...}

Nun ist es aber an der Zeit, ein paar Beispiele solch eleganter Beweise zu präsentieren. Der Klassiker schlechthin ist Euklids Beweis der Unendlichkeit der Primzahlen. Eine Primzahl ist bekanntlich eine Zahl größer als 1, die nur durch sich selbst und 1 teilbar ist. Jeder kennt die ersten Primzahlen 2, 3, 5, 7, 11, 13, und heute kann man Primzahlen mit Millionen von Stellen konstruieren, aber auch die raffiniertesten Methoden liefern bis jetzt nur endlich viele. Gibt es vielleicht nur endlich viele? Nein, und der Beweis der Unendlichkeit, der Euklid zugeschrieben wird, ist von bestechender Einfachheit. Euklid argumentierte indirekt: Angenommen, es gäbe nur endlich viele Primzahlen, nennen wir sie $\mathrm{p}_{1}, \mathrm{p}_{2}, \ldots, \mathrm{p}_{\mathrm{k}}$. Dann bilden wir die neue $Z$ ahl $M=p_{1} \times p_{2} \times \ldots x p_{k}+1$. M hat einen Primteiler $p$, und dieser Teiler muss nach unserer Annahme unter den $p_{i}$ vorkommen. Damit teilt $p$ die Zahl M, das Produkt $\mathrm{p}_{1} \mathrm{x} \ldots \mathrm{x} \mathrm{p}_{\mathrm{k}}$, also auch die Differenz 1. Die Zahl 1 hat aber keine Primteiler! - Widerspruch und Ende des Beweises.

Es gibt Dutzende weiterer amüsanter und lehrreicher Beweise für diesen Satz, aber der euklidische ist ein Muster an Transparenz und Leichtigkeit, und er arbeitet auch die Gründe für die Unendlichkeit heraus. Sieht man sich die Argumentation genau an, so erkennt man, dass sie auf zwei Tatsachen beruht: Es gibt unendlich viele Zahlen, 1, 2, 3, 4, .., und jede Zahl größer als 1 besitzt einen Primteiler. Man kann also einen analogen Schluss auf jedes algebraische System anwenden, das denselben Bedingungen genügt.

Thomas von Randow alias Zweistein, der jahrzehntelang die Wissenschaftsredaktion der Zeit leitete, brachte einmal in einer Augustnummer den Beweis von Euklid und resümierte ihn kurz: So viele Primzahlen ich auch habe, es gibt stets noch eine weitere, also unendlich viele. Wie immer erhielt er eine Anzahl von Zuschriften, darunter auch von einer Hausfrau in Bayern, die ihm schrieb: "Sehr geehrter Herr von Randow, ich sitze hier am Ammersee inmitten einer Heerschar von Mücken. So viele ich auch erschlage, es gibt immer noch eine weitere, kann ich daher schließen ...?« Ja, ein Journalist hat es auch nicht leicht.

Mit Unendlichkeiten ganz anderer Art befasste sich Georg Cantor, der Schöpfer der Mengenlehre. Seine ra- dikale Neubegründung der Mathematik wurde ebenso kompromisslos von vielen seiner Zeitgenossen abgelehnt, so dass der große David Hilbert ihm mit dem Ruf zu Hilfe eilen musste: "Niemand wird uns aus dem Paradies vertreiben, das Cantor für uns geschaffen hat!« Eine seiner größten Leistungen betraf die Frage: Wann sind zwei Mengen gleich groß? Für endliche Mengen bereitet dies natürlich kein Problem: Wir zählen sie einfach ab, und wenn dieselbe Anzahl herauskommt, dann (und nur dann) sind die Mengen gleich groß. Aber wie ist es mit unendlichen Mengen? Gibt es verschieden große Unendlichkeiten? Machen wir das folgende Gedankenexperiment: Nehmen wir an, einige Personen steigen in einen Bus; können wir feststellen, ob die Anzahl der Leute gleich jener der Sitze ist, ohne die Personen zu zählen? Natürlich geht das: Der Busfahrer ruft "Setzen«, und falls jede Person einen Sitz findet und kein Sitz frei bleibt, dann sind die beiden Mengen (der Leute und der Sitze) gleich groß. Mit anderen Worten, die beiden Mengen sind gleich groß, wenn es eine eindeutige Zuordnung oder, wie die Mathematiker sagen, eine Bijektion zwischen ihnen gibt.

Diese Idee übertrug Cantor nun auf ganz beliebige Mengen A und B: Sie sind gleich groß, wenn es eine Bijektion von A auf B gibt. Für endliche Mengen entspricht dies, wie gesehen, genau unserem gewohnten Zählbegriff, aber für unendliche Mengen wird Cantors Theorie sehr interessant und in hohem Maße nicht-intuitiv. Nehmen wir zum Beispiel die Menge $N=\{1,2,3,4, \ldots\}$ der natürlichen Zahlen. Wir nennen eine beliebige Menge A abzählbar, falls sie bijektiv auf $\mathrm{N}$ abgebildet werden kann, oder anders ausgedrückt, wenn die Elemente von $\mathrm{A}$ in der Form $\mathrm{a}_{1}, \mathrm{a}_{2}, \mathrm{a}_{3}, \ldots$ durchnummeriert werden können. Aber jetzt passiert etwas Unerwartetes. Angenommen, wir geben zu A ein weiteres Element $\mathrm{z}$ hinzu. Dann ist A zusammen mit $z$ immer noch abzählbar und daher gleich groß wie A, obwohl doch A in der Menge A plus z enthalten ist! Eine hübsche Illustration für dieses merkwürdige Phänomen ist >Hilberts Hotel : Es hat abzählbar viele Zimmer mit den Nummern 1, 2, 3, 4 und so weiter, und es ist vollkommen ausgebucht. Nun kommt ein neuer Gast an und verlangt ein Zimmer, worauf der Hotelmanager sagt: Tut mir Leid, alle Zimmer sind belegt. Kein Problem, sagt der Gast: Bitten Sie doch den Gast aus Zimmer 1, in Zimmer 2 zu übersiedeln, den aus Zimmer 2 in Zimmer 3, und so fort - und dann nehme ich das frei gewordene Zimmer 1. Zur Überraschung des Managers 


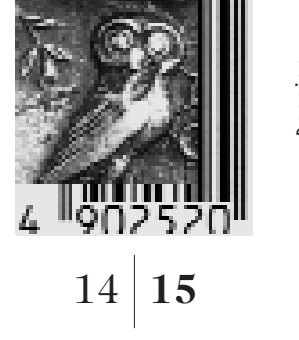

(er ist des Unendlichen nicht so recht mächtig) funktioniert das: Er kann wieder alle Gäste unterbringen und den neuen Gast dazu.

\section{... zum Unvollständigen}

Verlassen wir >Hilberts Hotek und sehen wir uns ein paar vertraute $Z$ ahlbereiche an. Die Menge $Z$ der ganzen Zahlen (positiv, negativ und 0) ist wieder abzählbar, da wir $Z$ in der Form $Z=\{0,1,-1,2,-2, \ldots\}$ durchnummerieren können. Auch die Menge aller Brüche ist nach wie vor abzählbar. Und wie ist es mit den reellen Zahlen R? Hier konnte Cantor mit einer genialen Idee zeigen, dass $\mathrm{R}$ nicht mehr abzählbar ist. Wie schon Euklid argumentierte Cantor indirekt. Betrachten wir die reellen Zahlen zwischen 0 und 1 . Jede solche Zahl $\mathrm{r}$ ist ein nicht endender Dezimalbruch $r=0, r_{1} r_{2} r_{3} \ldots$ so wie etwa $1 / 3=$ 0,33333... Angenommen, diese Zahlen könnten nummeriert werden, dann schreiben wir sie in einem Schema untereinander:

$$
\begin{aligned}
& \mathrm{r}_{1}=0, \mathrm{r}_{11} \mathrm{r}_{12} \mathrm{r}_{13} \ldots \\
& \mathrm{r}_{2}=0, \mathrm{r}_{21} \mathbf{r}_{22} \mathrm{r}_{23} \ldots \\
& \mathrm{r}_{3}=0, \mathrm{r}_{31} \mathbf{r}_{32} \mathbf{r}_{33} \ldots
\end{aligned}
$$

Die >Diagonale $<$ des Schemas ist hervorgehoben, also die Zahlen $r_{11}, r_{22}, r_{33} \ldots$ Wir wählen nun für jeden Index $n$ eine Kommastelle $b_{n} \neq 0$, welche verschieden von $r_{n n}$ ist (offensichtlich ist dies möglich), und bilden $b=$ $0, b_{1} b_{2} b_{3} \ldots$ Da die Zahl b zwischen 0 und 1 liegt, muss sie in unserer Liste vorkommen und hat daher einen Index, sagen wir $b=r_{k}$. Aber das geht nicht, $d a b_{k} \neq r_{k k}$ ist - und das ist der ganze Beweis!

Diese verblüffende Diagonalisierungsmethode von Cantor ist zu Recht in den Olymp der Mathematik aufgestiegen: Kürzer und eleganter geht es wirklich nicht! Und was die Stringenz betrifft, so führt sie uns bis an die Wurzeln der mathematischen Erkenntnis. Mit einer ganz ähnlichen Diagonalisierung bewies Kurt Gödel seinen Unvollständigkeitssatz, der von vielen als das bedeutendste mathematische Ergebnis des letzten Jahrhunderts angesehen wird. Dabei geht es um Folgendes: Gödel zeigte, dass es in jedem formalen System, in dem wir Schlüsse aufgrund unserer logischen Regeln ziehen, immer Sätze gibt, die innerhalb dieses Systems nicht bewiesen werden können. Und jetzt kommt die Pointe: Einer dieser Sätze ist die Widerspruchsfreiheit der Mathematik selbst! Wir werden niemals wissen, ob die Mathematik, wie wir sie betreiben, nicht einen Widerspruch in sich enthält und alles wie ein Kartenhaus zusammenstürzt.

Und wenn es denn so ist, dass die Mathematik eine pure Ausgeburt des Gehirns ist (es ist ja alles in der Mathematik erdacht), sie aber möglicherweise widerspruchsvoll ist, liegt da nicht der Rückschluss nahe, dass unser Gehirn vielleicht nicht ganz richtig verdrahtet ist oder dass es ganz anders funktionieren könnte und damit auch eine ganz andere Mathematik möglich und denkbar ist? Der Unvollständigkeitssatz ist sozusagen die Erbsünde der Mathematik. Wie Katholiken um die Erbsünde wissen (und sich nicht weiter darum kümmern), so wissen auch die Mathematiker um den schwankenden Grund, auf dem sie sich bewegen: Sie schreiben trotzdem ihre Bücher und beweisen ihre Sätze, immer in der Hoffnung, dass ihre Theoreme dereinst Eingang finden in das Paradies der ewigen Wahrheiten - und der eleganten Beweise.

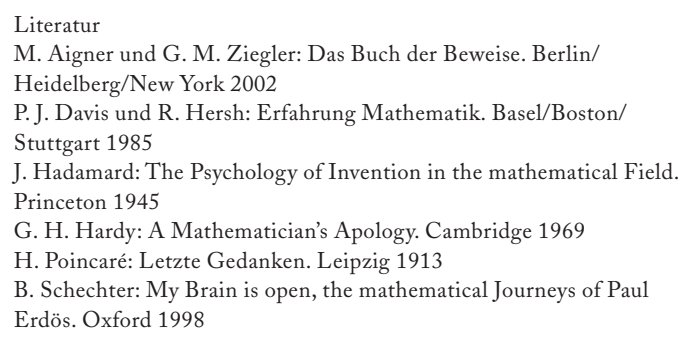

